\title{
ARTícUlO \\ Nueva descripción de Cossura chilensis Hartmann- Schröder, 1965 (Polychaeta: Cossuridae), de la Bahía de Valparaíso, Chile central con notas de su ecología
}

\author{
New description of Cossura chilensis Hartmann-Schröder, 1965 \\ (Polychaeta: Cossuridae), from Valparaíso Bay, central Chile \\ with notes of its ecology
}

\section{Eulogio H. Soto ${ }^{1}$ y Diego Lemus ${ }^{1}$}

\begin{abstract}
${ }^{1}$ Facultad de Ciencias del Mar y de Recursos Naturales, Universidad de Valparaíso, Avenida Borgoño 16344, Reñaca, Viña del Mar, Chile. eulogio.soto@uv.cl

Abstract.- Cossura chilensis is one of the most abundant polychaete species on soft-bottom benthic communities in central Chile. Nevertheless, it was firstly described by Hartmann-Schröder in 1965 and re-described by Carrasco in 1977; both studies with incomplete specimens. The aim of this research was to made a new description of the species in a detailed way from entire specimens using Scanning Electronic Microscopy. Cossura chilensis has a conical prostomium with a dorsal furrow and its peristomium is short and split by transverse ring. The first chaetiger is uniramous and the following ones are biramous. The branchial filament arises from the anterodorsal part of third chaetiger. Chaetae include short capillaries in the 3 first chaetigers; long capillaries in both rami along the rest of the body and thick capillaries in neuropodia of chaetigers 2-10. In addition, short spine-like chaetae are present from posterior segments. All chaetae are hirsute presenting a dense feather-appearance just by one side of the chaetae. Thoracic segments are short and crowded, while abdominal segments are longer than broad and less crowded. The pygidium is small and round-tip with 3 long cirri around anus. Methyl green staining is intense in prostomium, less intense in peristomium, first two chaetigers and in lateral patches from third chaetiger. The highest mean abundance by field work was 897 ind. $\mathrm{m}^{-2}$ in September 2013 being a dominant species of the soft-bottom Valparaiso bay, central Chile.
\end{abstract}

Key words: Taxonomy, polychaete, annelids, Southeast Pacific

\begin{abstract}
Resumen.- Cossura chilensis es una de las especies más abundantes de poliquetos en las comunidades bentónicas de fondos blandos de Chile central. Sin embargo, fue descrita originalmente por Hartmann-Schröder en 1965 y redescrita por Carrasco en 1977; ambos estudios con ejemplares incompletos. El objetivo de este trabajo fue realizar una nueva descripción de la especie, en detalle y a partir de ejemplares enteros, usando Microscopia Electrónica de Barrido. Cossura chilensis tiene un prostomio cónico con un pliegue dorsal y su peristomio es corto y está divido por un anillo transversal. El primer segmento setígero es unirrámeo mientras que los siguientes son birrámeos. El filamento branquial surge desde la parte anterodorsal del tercer setígero. Los ejemplares presentan setas capilares cortas en los primeros 3 setígeros, setas capilares largas en las dos ramas de la mayor parte del cuerpo y setas capilares gruesas en los neuropodios de los segmentos 2-10. Adicionalmente, están presentes setas cortas con forma de espina en los segmentos posteriores. Todas las setas son hirsutas presentando un aspecto densamente plumoso en un sólo lado de la seta. Los segmentos torácicos son cortos y bien agrupados, mientras que los del abdomen son más largos que anchos y menos agrupados. El pigidio es pequeño y redondeado con 3 cirros largos alrededor del ano. El patrón de tinción con verde metilo muestra una intensa tinción del prostomio, en menor grado del peristomio y los 2 primeros setígeros y la formación de parches laterales desde el tercer setígero. La mayor abundancia promedio por campaña fue de 897 ind. $\mathrm{m}^{-2}$ en septiembre 2013, constituyéndose en una especie dominante de los fondos sedimentarios de la Bahía de Valparaíso, Chile central.
\end{abstract}

Palabras clave: Taxonomía, poliquetos, anélidos, Pacífico sureste

\section{INTRODUCCIÓN}

Los representantes de la familia Cossuridae Day, 1963 son anélidos poliquetos de tamaño pequeño, que usualmente no superan los $15 \mathrm{~mm}$ de longitud. Son excavadores del fondo marino, habitando sustratos mixtos de arena y fango, pudiendo ser el grupo dominante de sus comunidades. Su morfología no es muy notoria y casi sin características externas que permitan su identificación, a excepción de un filamento branquial único, inserto en la parte dorsal y que emerge entre los segmentos 2- 
5 (Zhadan 2015). El segmento y la parte de éste en que el filamento se inserta, es una de las principales características taxonómicas a nivel de especie, junto con la forma del prostomio, cantidad y forma de los segmentos de las dos regiones corporales (tórax y abdomen), la disposición y tipo de setas a lo largo del cuerpo, la morfología del pigidio y el patrón de tinción (Hilbig 1996). La familia Cossuridae Day, 1963 consta de 26 especies, todas pertenecientes al género Cossura Webster \& Benedict, 1887 y algunas recientemente descritas en aguas adyacentes a Australia (Zhadan 2015). Anteriormente se aceptaban otros dos géneros: Cossurella Hartman, 1976 y Heterocossura Wu \& Chen, 1977. Este último fue reconocido como sinónimo de Cossurella, el que fue posteriormente aceptado como Cossura por Gardiner \& Wilson (1979). En Chile se han reportado tres especies pertenecientes al género Cossura (Rozbaczylo 1985), todas ellas descritas a partir de un bajo número de individuos incompletos. Estas corresponden a C. chilensis HartmannSchröder, 1965, C. alba Hartman, 1967 y C. abyssalis Hartman, 1967, encontradas a profundidades de 160, 957 y $3655 \mathrm{~m}$ de profundidad, respectivamente $(\operatorname{Read} 2000)$. La especie $C$. chilensis se distribuye desde Perú hasta Punta Lavapié, región de Biobío, Chile central y entre los 50-840 m de profundidad (Hartmann-Schröder 1965, Gutiérrez et al. 2003, Gallardo et al. 2004), encontrándose entre las especies de poliquetos más abundantes de las comunidades macrobentónicas de Chile central (Oyarzún et al. 1987, Gutiérrez et al. 2000, Gallardo et al. 2004, Sellanes et al. 2007) y siendo un miembro dominante en fondos blandos de la Bahía de Valparaíso (Soto et al. 2016). Fue originalmente descrita por Hartmann-Schröder (1965), quedando depositado el holotipo en el Museo Zoológico de Hamburgo, Alemania (Read \& Fauchald 2016) y posteriormente re-descrita por Carrasco (1977) como Cossura cf. chilensis, utilizando individuos incompletos cortados a la altura del tórax, recolectados en la Bahía de Concepción (Gallardo et al.1972) y omitiéndose por lo tanto rasgos relevantes de su morfología externa. El objetivo del presente trabajo es proveer una nueva descripción completa y detallada de la especie usando ejemplares enteros, incluyendo caracteres taxonómicos relevantes no antes descritos como el detalle de las setas, filamento branquial, parte posterior, pigidio y el patrón de tinción, así como también información de la ecología de la especie.

\section{MATERIALES Y MÉTODOS}

\section{RECOLECCIÓN DE EJEMPLARES}

Los ejemplares fueron recolectados entre noviembre 2012 y septiembre 2013, utilizando una draga van Veen modificada de $0,04 \mathrm{~m}^{2}$ de área de mordida, en 5 estaciones de muestreo en la Bahía de Valparaíso (estaciones 1-5), entre 25 y 140 m de profundidad (Fig. 1, Tabla 1), zona adyacente a Punta Curaumilla que es la localidad tipo. Las muestras de sedimento (3 réplicas por estación) fueron llevadas al Laboratorio de Bentos de la Facultad de Ciencias del Mar y de Recursos Naturales de la Universidad de Valparaíso, donde fueron tamizadas a $500 \mu \mathrm{m}$, separando a los ejemplares en frascos y conservándolos en alcohol diluido al 70\%. Los especímenes fueron observados en un microscopio estereoscópico Nikon® SM2800 y en un microscopio óptico Olympus ${ }^{\circledR}$ CX31 provisto de cámara clara, con la que se realizaron los esquemas. Para resaltar caracteres taxonómicos relevantes algunos individuos fueron sumergidos durante un minuto en una solución de verde metilo diluida con agua colocada en una placa de vidrio. Los individuos fueron además medidos y fotografiados con el software Micrometrics LE, utilizando un microscopio óptico Nikon® Eclipse E 200 provisto de una cámara fotográfica. La estimación de abundancia consistió en contar cada individuo de cada réplica para posteriormente extrapolarse los datos a un área de $1 \mathrm{~m}^{2}$. Con esta información también se calculó la desviación estándar.

Tabla 1. Datos de las estaciones muestreadas: Posición geográfica, profundidad y composición del sustrato / Data of sampled stations: Geographic position, depth and substrate

\begin{tabular}{cccccc}
\hline Estación & Latitud S & Longitud W & Profundidad (m) & \% Arena & $\%$ Fango \\
\hline 1 & $32^{\circ} 54^{\prime} 31.88^{\prime \prime}$ & $71^{\circ} 32^{\prime} 4.75^{\prime \prime}$ & 25 & 46,9 & 53,1 \\
2 & $32^{\circ} 54^{\prime} 31.91^{\prime \prime}$ & $71^{\circ} 34^{\prime} 49.16^{\prime \prime}$ & 50 & 48,0 & 52,0 \\
3 & $32^{\circ} 54^{\prime} 31.91^{\prime \prime}$ & $7^{\circ} 35^{\prime} 44.94^{\prime \prime}$ & 80 & 47,0 & 53,0 \\
4 & $32^{\circ} 54^{\prime} 31.92^{\prime \prime}$ & $71^{\circ} 36^{\prime} 48.08^{\prime \prime}$ & 100 & 46,0 & 54,0 \\
5 & $32^{\circ} 54^{\prime} 31.94^{\prime \prime}$ & $71^{\circ} 39^{\prime} 57.70^{\prime \prime}$ & 140 & 60,0 & 40,0 \\
\hline
\end{tabular}




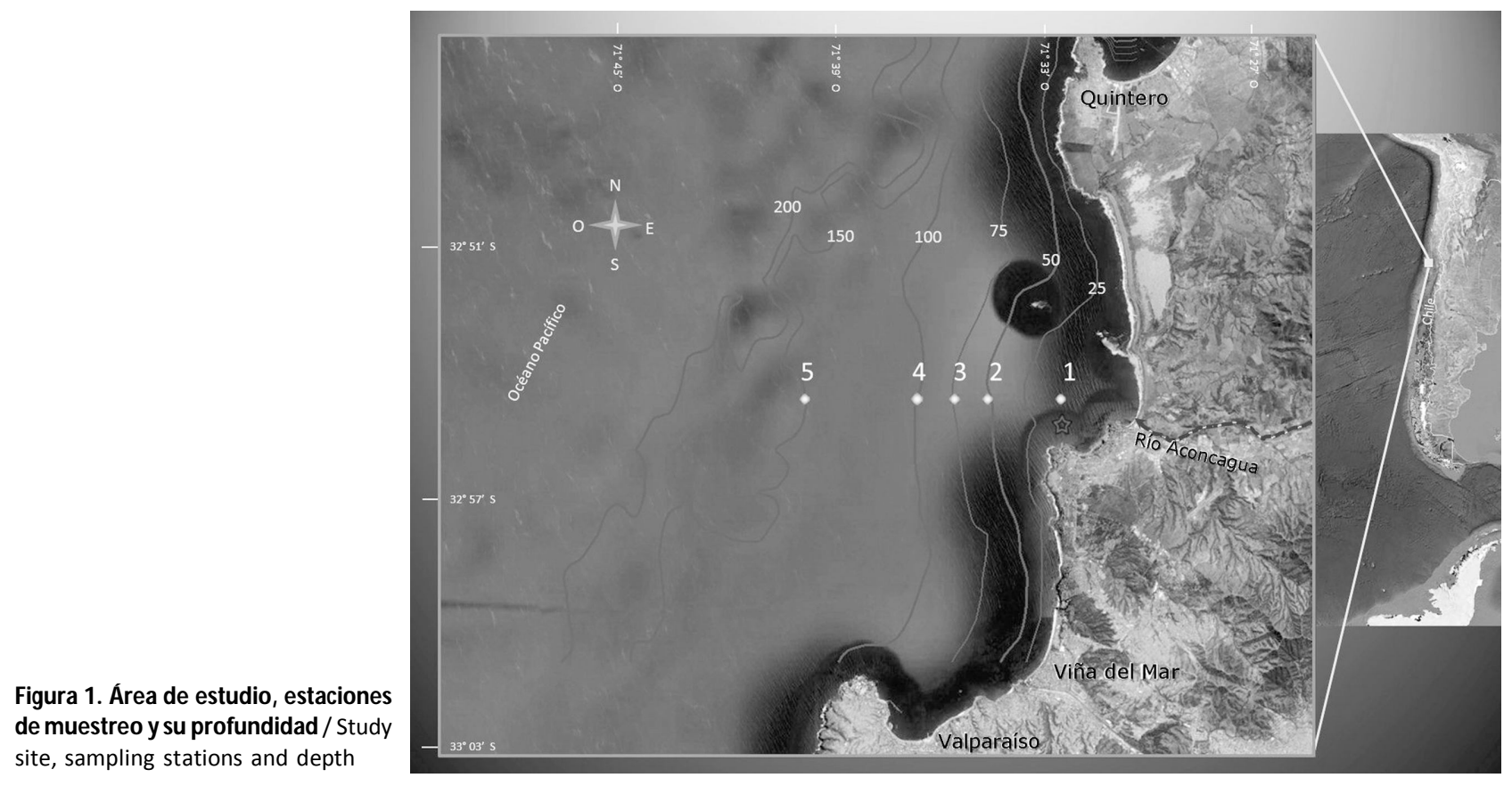

\section{Microscopia Electrónica de Barrido}

Se seleccionaron ejemplares completos y en buen estado para el pre-tratamiento y preparación de las muestras. Este proceso fue realizado en el Servicio de Microscopía Electrónica de Barrido de la Universidad Católica del Norte, Campus Guayacán, Coquimbo. Las muestras fueron fijadas en glutaraldehído al $2 \%$ en tampón cacodilato según la técnica descrita por Turner \& Boyle (1975). A continuación fueron deshidratadas en concentraciones crecientes de etanol hasta llegar a etanol absoluto y secadas mediante punto crítico con $\mathrm{CO}_{2}$ líquido como líquido intermediario en un secador Samdri780A Tousimis. Posteriormente, se montaron sobre soportes cilíndricos de bronce con un adhesivo de doble faz. Finalmente se cubrieron con oro en una sombreadora Fine Coat Ion Sputter J.F.C.-100 JEOL y se observaron en un microscopio electrónico de barrido Carl Zeiss ${ }^{\circledR}$ modelo EVO MA 10 para su digitalización y procesamiento de imágenes en el Departamento de Física de la Universidad Técnica Federico Santa María, Valparaíso.

\section{GRANULOMETRía Y TEMPERATURA}

Las muestras de sedimento (200 g) para el estudio granulométrico fueron obtenidas desde cada estación de muestreo con una draga van Veen de $0,04 \mathrm{~m}^{2}$ de área de mordida. Se determinó el tamaño de grano del sedimento utilizando un agitador magnético con 6 tamices con diámetros de apertura de $4000 \mu \mathrm{m}$ para conchuela y grava, $2000 \mu \mathrm{m}$ para arena medianamente gruesa, $1000 \mu \mathrm{m}$ para arena gruesa, $500 \mu \mathrm{m}$ para arena media, $250 \mu \mathrm{m}$ para arena fina, 125 para arena medianamente fina y $63 \mu \mathrm{m}$ para limo grueso. Las muestras se secaron en una estufa Memmert a $50^{\circ} \mathrm{C}$ para luego tamizarlas en un agitador mecánico JEL 4188 por 15 min. Los sedimentos se clasificaron con respecto al tamaño de grano de acuerdo a la escala de Folk \&Ward (Blott \& Pye 2001). Las mediciones de temperatura fueron realizadas con un CTD Seabird 19 plus.

\section{Resultados}

Familia Cossuridae Day, 1963

Género Cossura Webster \& Benedict, 1887

Cossura chilensis Hartmann-Schröder, 1965 Figs. 2-5.

Cossura chilensis Hartmann-Schröder, 1965: 223-224, figs. 216-218. Carrasco 1977: 82-84, figs. 34-42.

\section{Material eXaminado:}

Campaña 1 (98 ejemplares): Estaciones: 2(4), 3(45), 5(49). 30 de noviembre y 1 de diciembre 2012.

Campaña 2 (39 ejemplares): Estaciones: 3 (16), 4 (20), 5 (3). 19 y 20 de marzo 2013

Campaña 3 (480 ejemplares): Estaciones: 3 (191), 4 (81), 5 (208). 10 y 11 de junio 2013

Campaña 4 (537 ejemplares): Estaciones: 3 (157), 4 (353), 5 (27). 4 y 5 de septiembre 2013 
Campaña 5 (549 ejemplares): Estaciones: 2 (2), 3 (249), 4 (87), 5 (211). 10 y 12 de diciembre 2013.

Información de posición, profundidad y granulometría de las estaciones de muestreo se detalla en la Tabla 1.

\section{NuEVA DESCRIPCIÓN}

Especímenes observados de coloración pálida en alcohol y sin manchas oculares. El mayor espécimen (neotipo) midió 16,3 mm de longitud y 0,47 mm de ancho en el tórax, con un total de 75 setígeros (Figs. 2a y 4a). Prostomio triangular, redondeado o puntiagudo en su extremo, más largo que ancho y ligeramente aplanado dorso-ventralmente; usualmente con un pronunciado pliegue dorsal (Figs. 2b, 3b, 4b y 4c). No se observaron órganos nucales. Peristomio al menos 2 veces más corto que el prostomio y dividido por un surco o anillo transversal (Fig. 2b). La cavidad bucal se abre entre estos 2 segmentos, saliendo ocasionalmente como una faringe tentaculada, formada por al menos 3 o 4 lamelas o tentáculos bucales (Fig. 3a), más frecuentemente como una bola fibrosa (Fig. 4b y 4c).

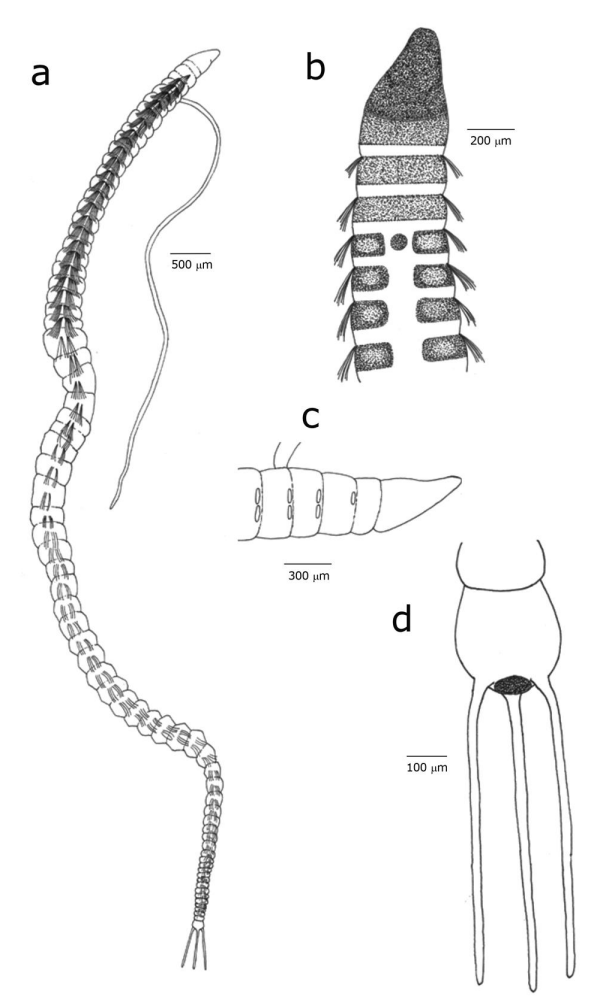

Figura 2. Cossura chilensis. a) cuerpo completo, vista lateral; b) patrón de tinción con verde metilo, parte anterior vista dorsal; c) inserción del filamento branquial, parte anterior vista lateral y d) detalle del pigidio, vista dorsal / Cossura chilensis: a) entire body, lateral view; b) methyl green staining pattern, anterior end dorsal view; c) insertion of branchial filament, anterior end lateral view and d) pygidium detail, dorsal view
Cuerpo relativamente robusto, con tórax aplanado y un marcado canal dorsal (Fig. 4c) desde atrás de la inserción del filamento branquial, en la parte anterior del tercer setígero (Figs. 2b-c, 3 b y 4c). Parte anterior del abdomen de apariencia lisa a rugosa, según estado de conservación y tan ancha comoel tórax, con segmentos el doble o más largos que los torácicos (Figs. 2 a y 5 e). Parte media del abdomen con segmentos más cortos y delgados. Parte posterior del abdomen más estrecha, con segmentos más cortos y bien agrupados (Fig. 5f).

Parapodios rudimentarios y sin gran variación a lo largo del cuerpo, aunque de forma trapezoidal pequeña en la parte posterior (Fig. 5b). Primer setígero unirrámeo, con setas más cerca del notopodio (Fig. 4b). Segmentos siguientes birrámeos. Tórax formado por entre 22 y 26 segmentos, según tamaño del animal, siendo 24 el número más común, mientras que el abdomen consta de 49 segmentos (Figs. 2a y 4 a). Filamento branquial dorsal surge de la parte central anterior del tercer setígero, pudiendo estar ligeramente desviado hacia la izquierda o hacia la derecha (Figs. 2a, c y 4b-c). Este filamento presenta un patrón de segmentación regular a lo largo y un surco longitudinal distal (Fig. 4d). No se observaron bandas ciliadas. La longitud del filamento branquial es variable llegando incluso hasta la mitad el cuerpo del animal. Su ancho puede alcanzar un tercio del tórax.

Setas en su mayoría capilares delgados de 4 tipos: a) capilares cortas, b) capilares largas, c) capilares gruesas y d) capilares con forma de espina. Las capilares cortas, más delgadas que las capilares largas, se encuentran exclusivamente en los primeros setígeros (1 a 3), en número de entre 2-3 (Figs. 4e y 4f), acompañadas por capilares largas en número de entre 4-5, sumando entre 6-7 setas para el primer setígero. Las capilares gruesas están en los neuropodios de los setígeros 2-10, en un número de entre 2-3, acompañadas por capilares largas entre 4-5, sumando 7 setas capilares por parapodio (Figs. 2d, 4eg). Las capilares largas ocupan exclusivamente los notopodios desde el setígero 2 en adelante y los neuropodios desde el setígero 11 en adelante (Figs. 4i, 5a). En el abdomen están presentes capilares largos, entre 2-4 por parapodio en el centro del setígero (Figs. 5c y 5d). Las capilares con forma de espina (Fig. 5b) se encuentran de 2-3 exclusivamente en el neuropodio del abdomen, son más cortas y robustas. Todas las setas, a excepción de los capilares cortos de los setígeros 1 a 3, son hirsutas y están cubiertas de finas vellosidades casi imperceptibles para la microscopía óptica. Estas vellosidades son de longitud variable y densamente plumosas en un sólo lado de la seta (Fig. 4h), apareciendo sobre la base de inserción del parapodio y ausente en el extremo de la seta (Figs. 4i, 5b-c). En capilares gruesos y cortos tipo espina las vellosidades se restringen a la zona media (Fig. 5b), mientras que en capilares largos de la 


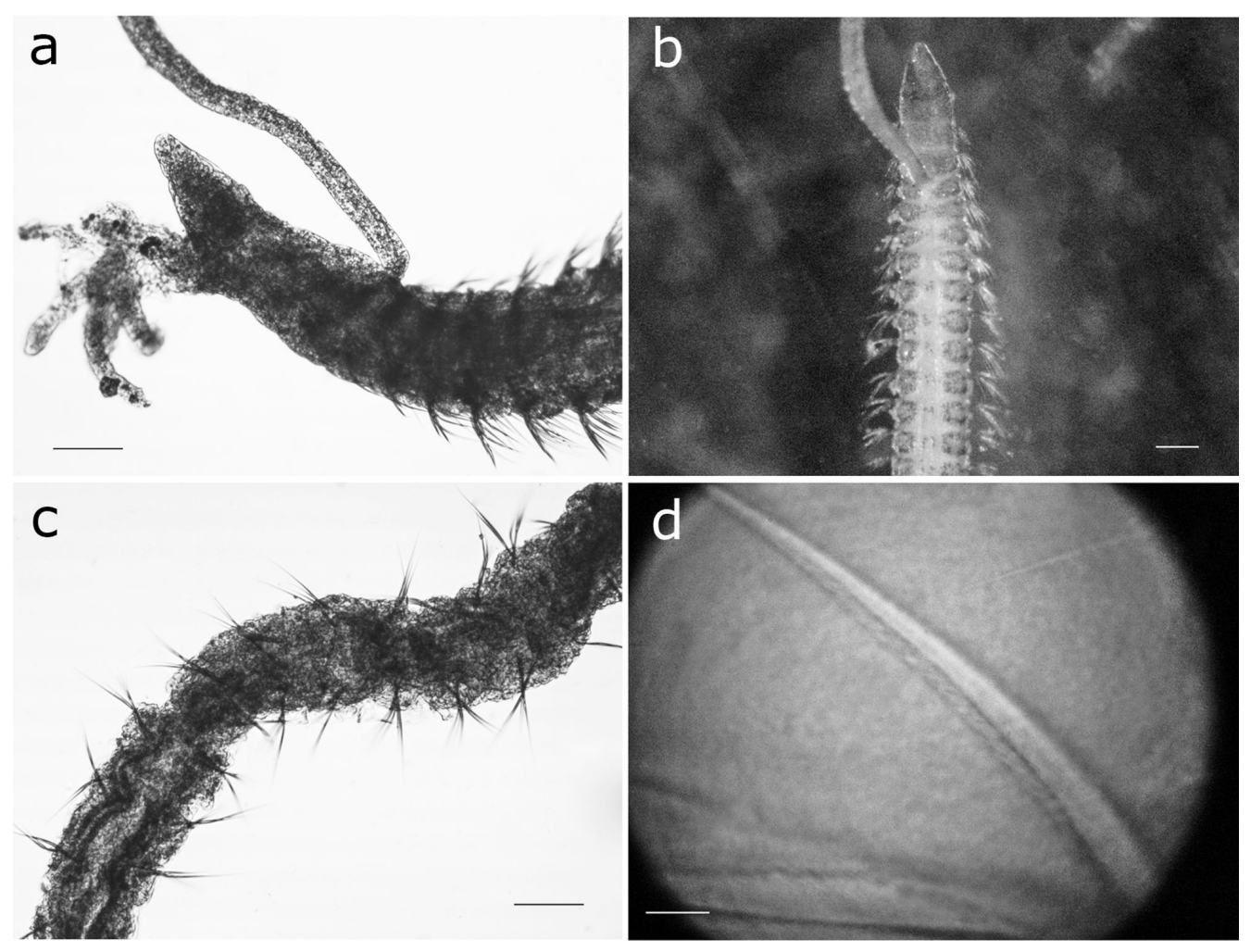

Figura 3. Cossura chilensis. a) estructura bucal, parte anterior, vista lateral; b) patrón de tinción con verde metilo, vista dorsal c) segmentos abdominales, vista dorsal; d) seta capilar parte anterior. Escala: a y c) $\mathbf{3 0 0} \mu \mathrm{m}$; b) $\mathbf{2 0 0} \mu \mathrm{m}$ y d) $\mathbf{1 0} \mu \mathrm{m}$. Microscopía óptica / Cossura chilensis. a) buccal structure, anterior end, lateral view; b) methyl green staining pattern, dorsal view; c) abdominal segments, dorsal view; d) capillary chaetae anterior end. Scale bars: a and c) $300 \mu \mathrm{m}$, b) $200 \mu \mathrm{m}$ and d) $10 \mu \mathrm{m}$. Light microscope

parte abdominal terminal (setígero 74, Fig. 5d) las vellosidades aparecen desde la base. En algunas setas esta apariencia plumosa no es densa, con vellosidades superficiales que asemejan largos dientes finos, como una seta aparentemente aserrada o limbada. No hay acículas a lo largo del cuerpo. Pigidio simple, pequeño y redondeado, precedido por entre 1 y 2 segmentos preanales asetígeros (Figs. 2 d y 5 g). Presenta 3 cirros delgados, largos y flexibles, ubicados de manera equidistante alrededor del ano y sin procesos intercirrales (Figs. $2 \mathrm{~d}$ y $5 \mathrm{~h})$.

\section{Patrón de tinción con Verde metilo}

Prostomio y filamento branquial teñidos intensamente. En todos los segmentos subsecuentes, incluyendo el peristomio, sólo se tiñen los primeros dos tercios de cada segmento. Setígeros 1 y 2, al igual que el peristomio, se tiñen además dorso-lateralmente, con menor intensidad que el prostomio. Desde el setígero 3 en adelante se forman parches laterales, densamente teñidos en los bordes y en menor grado hacia el centro de éstos, dejando así la región mediodorsal sin teñir (Figs. 2b y 3b). Este patrón de parches se mantiene a lo largo del tórax, desapareciendo en el abdomen.

\section{HÁBitat}

El sustrato registrado en cada estación correspondió a una mezcla de arena y limo grueso en una proporción cercana al $50 \%$, a excepción de la estación 5 (140 m), que presentó un $60 \%$ de arena y un $40 \%$ de limo grueso (Tabla 1 ). El rango de temperatura del fondo fue de entre 10,89 y $13,08^{\circ} \mathrm{C}$.

\section{ECOLOGÍA Y DISTRIBUCión BATIMÉTRICA}

La mayor abundancia promedio por estación correspondió a $1.048 \pm 1.279$ ind. $\mathrm{m}^{-2}$ en la estación $4(100 \mathrm{~m})$ y la mayor abundancia promedio por campaña correspondió a $897 \pm 1.266$ ind. $\mathrm{m}^{-2}$, en septiembre 2013, para un registro total de 9667 ind. $\mathrm{m}^{-2}$ en el área de estudio. La abundancia total de $C$. 

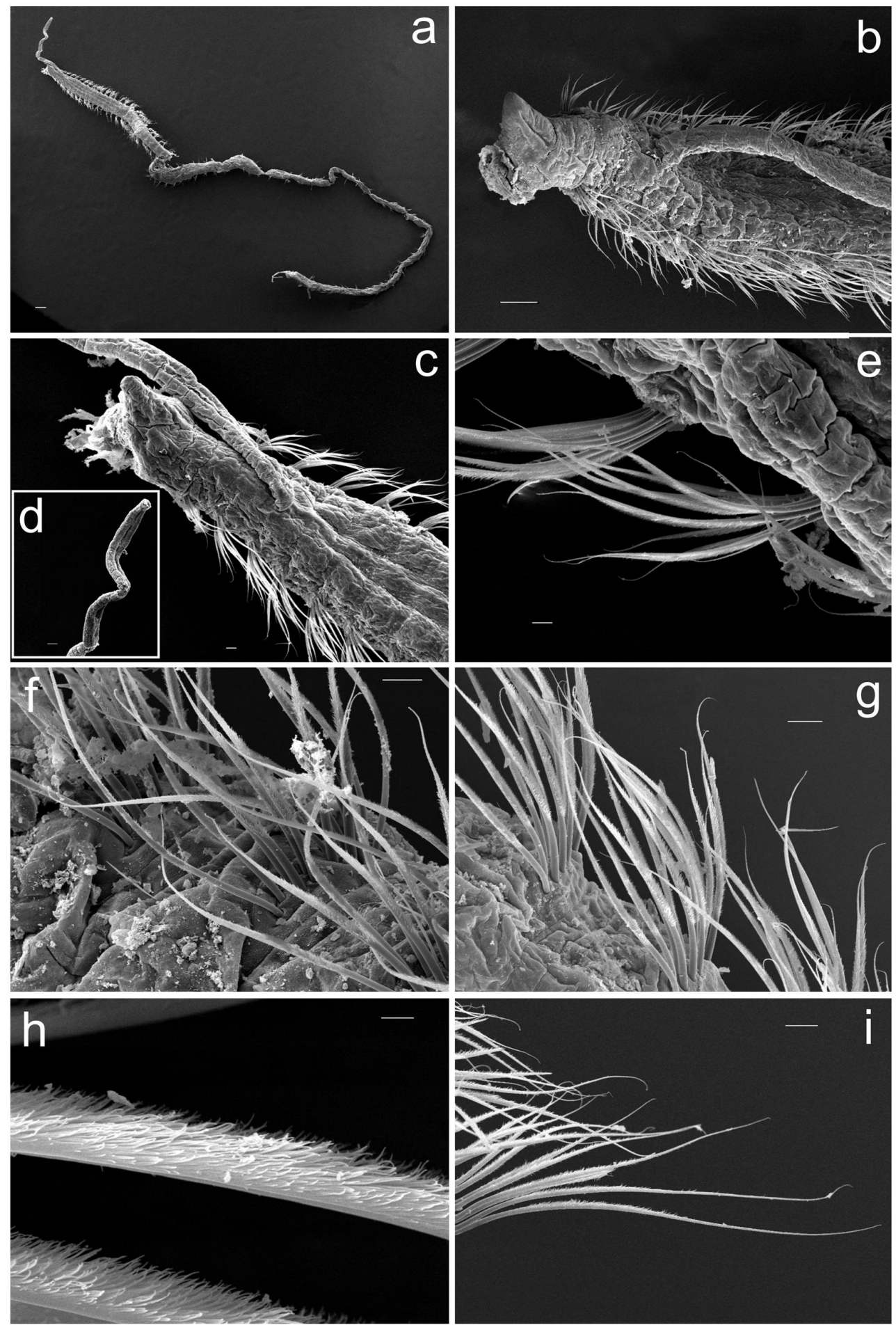

Figura 4. Cossura chilensis. Imágenes de microscopía electrónica de barrido. a) Cuerpo completo, vista dorsal; b) parte anterior vista dorso-lateral; c) parte anterior vista dorsal; d) detalle del filamento branquial; e) setas notopodiales en setígeros 1-3; f) setas de primeros setígeros; g) seta notopodial del setígero 5; h) detalle de la seta del setígero 1; i) setas del setígero 20 . Escala: a) $200 \mu \mathrm{m}$; b) $100 \mu \mathrm{m}$; c, d, f, g, i) $20 \mu \mathrm{m}$; e) $10 \mu \mathrm{m}$ y h) $2 \mu \mathrm{m} /$ Cossura chilensis. Scanning electron microscope images. a) entire body, dorsal view; b) anterior end dorso-lateral view; c) anterior end dorsal view; d) branchial filament detail; e) notochaetae of chaetigers 1-3; f) chaetae of first chaetigers; g) notochaetae of chaetiger 5 ; h) chaetae detail of chaetiger 1; i) chaetae of chaetiger 20. Scale bars: a) $200 \mu \mathrm{m}$; b) $100 \mu \mathrm{m}$; c, d, f, g, i) $20 \mu \mathrm{m}$; e) $10 \mu \mathrm{m}$ and h) $2 \mu \mathrm{m}$

614 Soto \& Lemus

Descripción de Cossura chilensis (Polychaeta) de la bahía de Valparaíso 

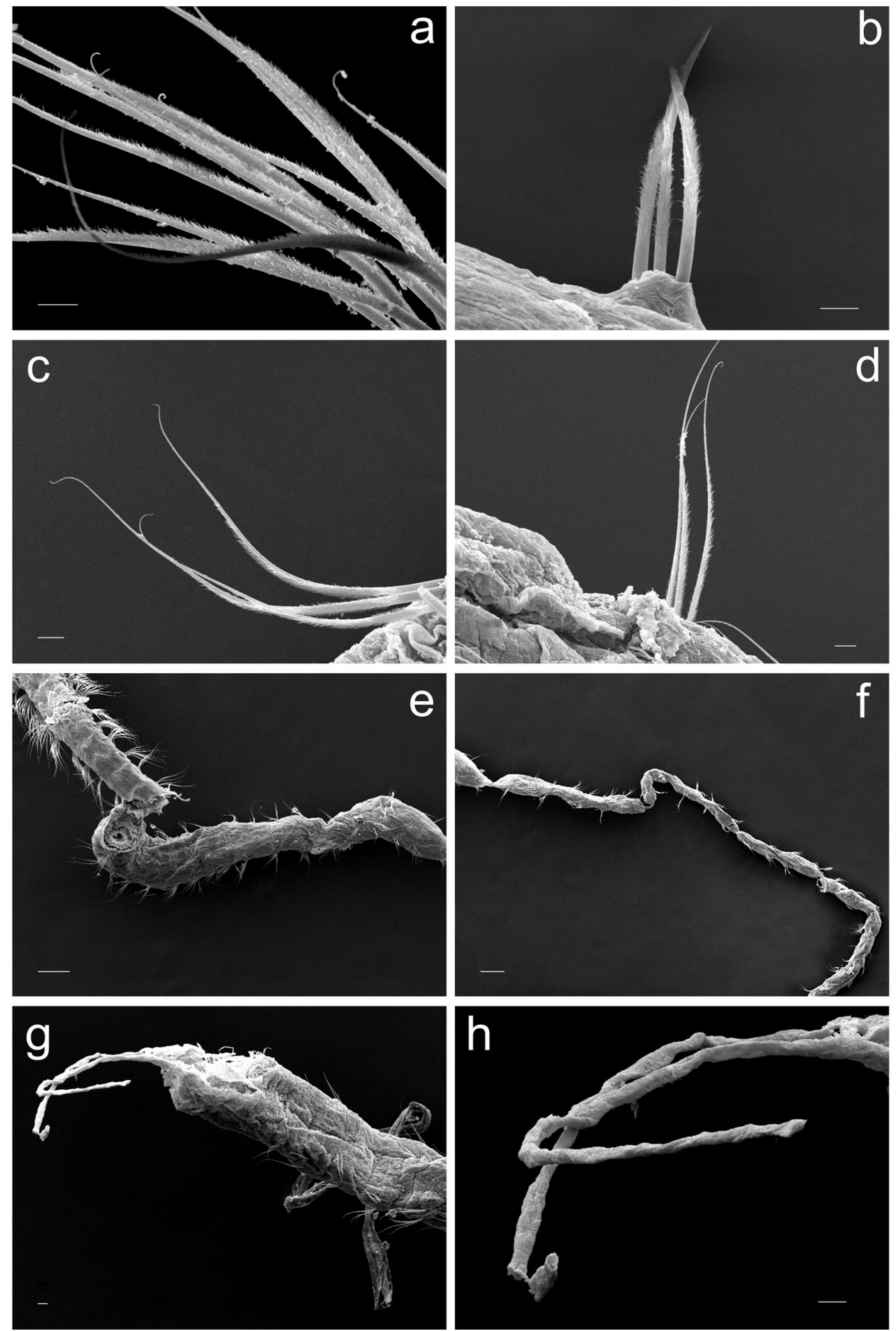

Figura 5. Cossura chilensis. Imágenes de microscopía electrónica de barrido. a) Setas neuropodiales del segmento 28; b) setas notopodiales del setígero 53 ; c) setas neuropodiales del setígero 66 ; d) setas notopodiales del setígero 74; e) parte media del cuerpo; f) región abdominal; g) región

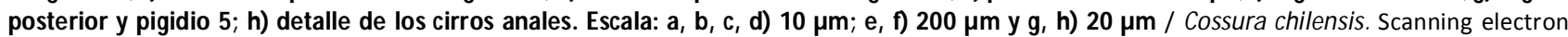
microscope images. a) neurochaetae of chaetiger 28 ; b) notochaetae of chaetiger 53 ; c) neurochaetae of chaetiger 66 ; d) notochaetae of chaetiger 74; e) middle part of the body; f) abdominal region; g), posterior region and pygidium; h) anal cirri detail . Scale bars: a, b, c, d) $10 \mu \mathrm{m}$; e, f) $200 \mu \mathrm{m}$ and $\mathrm{g}$, h) $20 \mu \mathrm{m}$ 


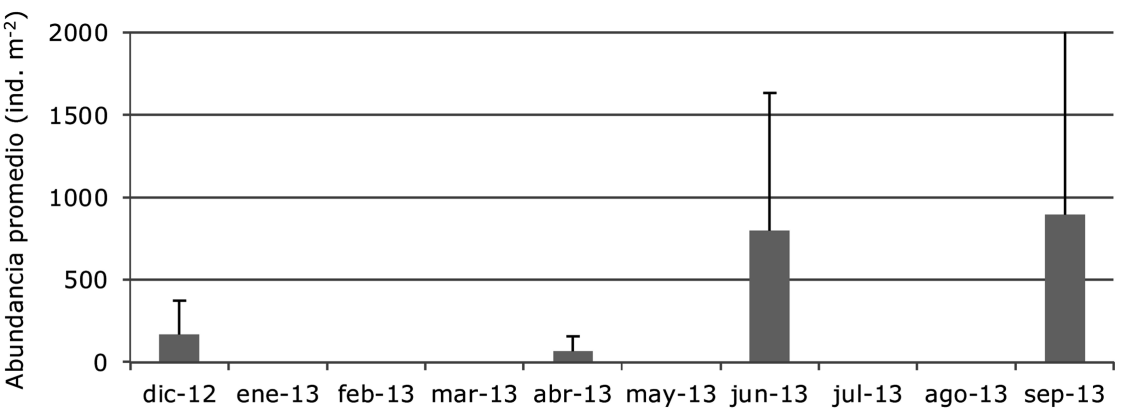

Campañas de muestreo

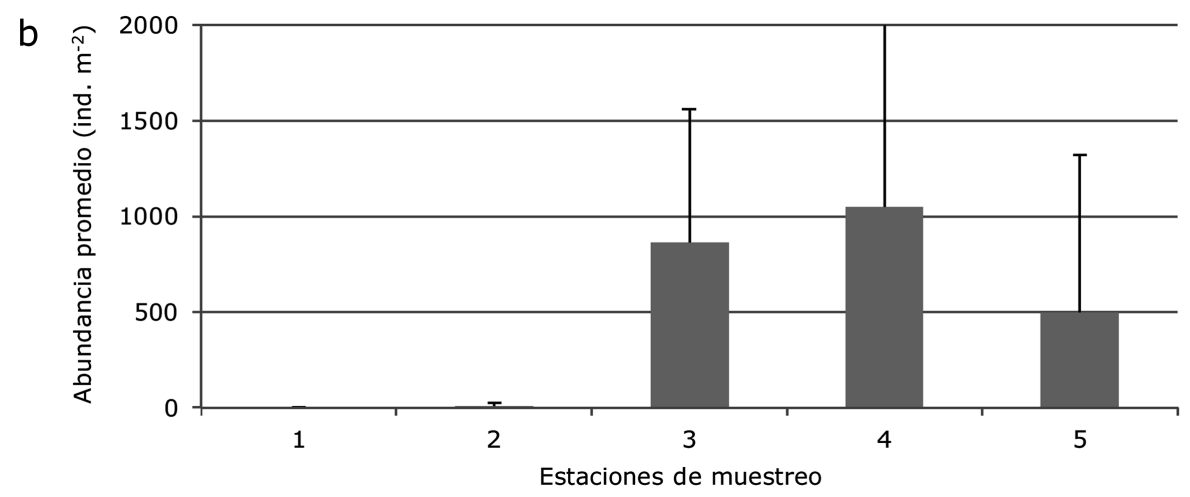

Figura 6. Variabilidad temporal (a) y espacial (b) de la abundancia promedio (ind. $\mathbf{m}^{-2}$ ) de Cossura chilensis en la zona de estudio / Temporal (a) and spatial (b) variability of mean abundance (ind. $\mathrm{m}^{-2}$ ) of Cossura chilensis at study site

chilensis registró su valor más alto en la estación 4, a 100 m de profundidad, durante la campaña realizada en septiembre 2013, con una abundancia de 2.942 ind. $\mathrm{m}^{-2}$. No se encontraron ejemplares de $C$. chilensis en la estación 1, a $25 \mathrm{~m}$ de profundidad, en ninguna de las campañas de muestreo; mientras que en la estación $2(50 \mathrm{~m})$ sólo se encontraron ejemplares de C. chilensis durante la campaña de diciembre 2012, con una abundancia total de sólo 33 ind. $\mathrm{m}^{-2}$. En las otras estaciones se registraron abundancias hasta dos órdenes de magnitud superiores, con promedios muy superiores en las campañas de junio y septiembre 2013 (Fig. 6).

\section{Discusión}

\section{TAXonomía}

La taxonomía de los cosúridos es compleja, debido por una parte a la escasez de características externas de discriminación y en segundo término a la existencia de causas de confusión. Una de estas causas es la creencia de que existen 2 segmentos peristomiales, siendo Thulin (1921) el primero en señalar que el llamado 'primer segmento peristomial' sería en realidad parte del prostomio. Hilbig (1996) también sugiere que en ocasiones la parte posterior del prostomio está separada por un anillo que es confundido con los dos primeros segmentos peristomiales. Por lo tanto el peristomio puede tener uno o dos segmentos asetígeros. Ewing (1984) analizó la segmentación peristomial y encontró que puede estar dorsal o ventralmente incompleta y que la línea segmental puede oscurecerse por la presencia de la boca o faringe. En $C$. chilensis un anillo transversal peristomial fue registrado en el presente estudio.

Otra causa de error ha sido el número de segmentos anteriores unirrameos, ya que al encontrarse los parapodios muy cerca entre si y lo rudimentario de éstos, ha llevado a describir hasta 8 segmentos anteriores unirrameos (Reish 1958). Sin embargo, hoy existe la convicción de la presencia de un único segmento unirrameo. Estas y otras discrepancias hacen que las re-descripciones de especies de la familia Cossuridae sean comunes en los últimos años, pudiendo citar ejemplos tales como las de C. chilensis por Carrasco (1977), de $C$. longocirrata Webster \& Benedict, 1887 por Fournier \& Petersen (1991), de C. pygodactylata Jones, 1956 por Zhadan et al. (2012) y la de $C$. consimilis Read, 2000 por Zhadan (2015). 
El prostomio cónico coincide con lo reportado tanto por Hartmann-Schröder (1965), como con la descripción realizada por Carrasco (1977). Este es un atributo común para toda la familia Cossuridae, con excepción de Cossura ginesi LiñeroArana \& Díaz-Díaz, 2010, que presenta un par de cuernos laterales bien desarrollados en la parte anterior del prostomio (Liñero-Arana \& Díaz-Díaz 2010). No obstante, los ejemplares examinados mostraron una variación en el extremo del prostomio desde una forma puntiaguda a redondeada, situación que también se ha observado en otras especies como $C$. longocirrrata y C. pygodactylata (Zhadan 2015). Los autores anteriores describen además, la existencia de 2 segmentos asetígeros, de los cuales el primero podría ser parte del prostomio. Este primer segmento peristomial incompleto podría deberse a la formación de un pliegue en el prostomio, producto de la contracción de los individuos (Thulin 1921). En los ejemplares observados se aprecia la división del peristomio por un anillo transversal dando lugar a un segmento asetígero (Figs. 2b y 4b). Carrasco (1977) describió además que la cavidad bucal se abre entre el prostomio y el peristomio. El aparato bucal que sale de dicha cavidad correspondería a una extensión de la pared dorsal de la faringe según Tzetlin (1994). En algunos ejemplares se observó que dicho aparato bucal estaría formadopor una faringe tentaculada (Fig. 3a) muy similar a la faringe observada en C. pygodactylata.

El primer segmento setígero unirrameo fue descrito para esta especie tanto por Hartmann-Schröder (1965) como por Carrasco (1977). Sin embargo las setas se mencionan saliendo del neuropodio y desde una posición central, respectivamente. Esto implicaría que la posición de las setas del primer setígero sería bastante variable o que depende en gran medida del ángulo en que se observa. En los ejemplares examinados se pudo apreciar que las setas del primer segmento aparecen generalmente desde el notopodio (Fig. 4b).

El filamento branquial emergiendo de la parte anterior del tercer segmento es también común en las otras descripciones realizadas para esta especie, sin embargo, refiriéndose a esta estructura como un tentáculo o palpo tentacular. Para este filamento no se observan bandas ciliadas como en $C$. pygodactylata (Zhadan et al. 2012.)

Tanto Carrasco (1977) como Hartmann-Schröder (1965), describen dos tipos de setas diferentes. El primero describe setas gruesas, entre los neuropodios 2-10 y setas delgadas aserradas en el resto del cuerpo, mientras que el segundo describe setas largas dentadas y setas cortas que se encuentran entre los setígeros 1-9. La existencia de 4 tipos de setas descritas en el presente trabajo correspondería parcialmente con las descripciones anteriores, pues las setas gruesas se encuentran presentes entre los setígeros 2-10, como señala Carrasco (1977) y las setas cortas descritas por Hartmann-Schröder (1965) entre los setígeros 1-9 corresponderían a las setas gruesas más las setas capilares cortas del primer segmento setígero. Las setas capilares largas, que se encuentran en la mayor parte del cuerpo, son descritas por ambos autores como de borde aserrado, mientras que en el presente trabajo se clarificó que son hirsutas (vellosidades densamente plumosas), gracias al empleo de microscopia electrónica de barrido. Estas setas son similares a las setas capilares posteriores descritas para $C$. ginesi (Liñero-Arana \& Díaz-Díaz 2010) y a las setas capilares anteriores descritas por Wu \& Chen (1977) para $C$. aciculata (Wu \& Chen, 1977). Carrasco (1977) describió además que las setas, tanto gruesas como delgadas, presentarían ornamentaciones pilosas, especialmente en la parte distal, lo cual no coincide con la descripción original ni con la presente ya que estas ornamentaciones no fueron observadas. Las setas presentan vellosidades muy finas, casi imperceptibles para la microscopía óptica, lo que explicaría que no hayan sido descritas originalmente, siendo necesaria la microscopía electrónica para detallar mejor su morfología.

La morfología del pigidio no ha sido anteriormente descrita para esta especie. El pigidio es pequeño y algo redondeado y presenta 3 cirros pigidiales largos y simples. Estos cirros se ubican de manera equidistante alrededor del ano y surgen aparentemente 2 de ellos desde dorsal y 1 desde ventral (Figs. $5 \mathrm{~g}$ y $5 \mathrm{~h}$ ). Este arreglo espacial se asemeja al de $C$. brunnea Fauchald, 1977, que al igual que $C$. chilensis, no presenta papilas interdigitales visibles, como se observan en $C$. pygodactylata (Hilbig 1996, Zhadan et al. 2012).

El patrón de tinción observado es característico de esta especie. Se asemeja en parte al de C. candida (Hartman 1955), en relación a la formación de parches laterales, aunque sin presentar su coloración clara en la punta del prostomio o en las líneas oblicuas del segmento anterior a la inserción del filamento branquial (Hilbig 1996).

\section{Ecología}

Cossura chilensis ha sido encontrada en sustrato mixto de arena y limo grueso, a una profundidad entre 50 y $140 \mathrm{~m}$ con un rango de temperatura entre 10,89 y $13,08^{\circ} \mathrm{C}$. HartmannSchröder (1965), por su parte, reportó diferentes sustratos para esta especie, como arena fina, limo de foraminíferos y detritos; siendo el sustrato típico la arena fina con piedras y algas muertas. El rango de profundidad fue entre 50 y $160 \mathrm{~m}$, con una temperatura no menor de $10,8^{\circ} \mathrm{C}$, lo que explicaría según la autora porqué $C$. chilensis no se encuentra presente en la zona sur, donde la temperatura es habitualmente menor. Estos 
antecedentes concuerdan con las características ambientales registradas en la Bahía de Valparaíso para el presente estudio. Sin embargo, el registro de densidades más altas a mayor profundidad y la total ausencia de individuos a $25 \mathrm{~m}$ (estación 1) indicarían que $C$. chilensis prefiere fondos más profundos con mayor proporción de fango, coincidiendo con lo registrado por Gallardo et al. (2004) para Bahía de Concepción. No obstante, Carrasco (1977), reporta en altas densidades a Cossura cf. chilensis a profundidades menores a $37 \mathrm{~m}$ en sustratos predominantes de fango negro. Adicionalmente, es importante destacar la capacidad de adaptación de esta especie a concentraciones variables de oxígeno disuelto. Según su rango de distribución batimétrica en los fondos sedimentarios de la zona de estudio ha sido registrada a concentraciones de oxígeno que varían desde $3 \mathrm{~mL} \mathrm{~L}^{-1}$ hasta valores $<0,5 \mathrm{~mL} \mathrm{~L}^{-1}$ propios de zonas de mínimo de oxígeno registradas en la Bahía de Valparaíso (Soto et al. 2016).

Las máximas abundancias fueron encontradas a $100 \mathrm{~m}$ de profundidad, en septiembre 2013, lo que indicaría que las mayores abundancias para esta especie se darían en primavera. Estas correspondieron a un valor cercano a los 3.000 ind. $\mathrm{m}^{-2}$. Gallardo et al. (2004) reportaron para Concepción, abundancias máximas a $122 \mathrm{~m}$ de profundidad para esta especie, correspondiente a 7.874 ind. $\mathrm{m}^{-2}$.
Según Read (2000), en Chile están presentes otras 2 especies pertenecientes al género Cossura, además de $C$. chilensis. Las otras especies, C.abyssalis Hartman, 1967 y C. alba Hartman, 1967, habitan a profundidades entre 3.655 y $957 \mathrm{~m}$, respectivamente (Hartman 1967) (Tabla 2). Además del punto de inserción del filamento branquial y de algunas mediciones poco representativas, nada se sabe sobre la taxonomía de estas especies, pues a pesar de existir descripciones, estas se basan en muy pocos especímenes y en individuos incompletos, evidenciando la necesidad de realizar re-descripciones de estas especies. Adicionalmente, existiría otra especie de Cossura diferente a $C$. chilensis (con mayor número de cirros pigidiales) presente en la región austral de Chile (Cañete, com. pers. $)^{1}$, sin embargo, no ha sido descrita.

Este trabajo representa una nueva descripción de esta especie realizada, esta vez, a partir de organismos completos, entregándose nuevos detalles de la morfología y caracteres diagnósticos obtenidos a partir del empleo de microscopia electrónica de barrido. En tal sentido se detallan las características de las setas, prostomio, filamento branquial, patrón de tinción con verde metilo, pigidio y la morfología general de la parte posterior del animal entre otros rasgos. Adicionalmente, se entrega información relevante respecto de la variabilidad temporal y espacial de su densidad en la bahía de Valparaíso.

Tabla 2. Comparación de algunas especies del género Cossura. Modificada de Read (2000) / Comparison of some species of Cossura genus. Modified from Read (2000)

\begin{tabular}{|c|c|c|c|c|c|c|}
\hline Especie & $\begin{array}{l}\text { Setígeros } \\
\text { anteriores }\end{array}$ & $\begin{array}{l}\text { Cirros } \\
\text { anales }\end{array}$ & $\begin{array}{l}\text { Números de especímenes, } \\
\text { dimensiones máximas } \\
(\mathrm{A}, \mathrm{L}) \text { y } \mathrm{N}^{\circ} \text { de segmentos }\end{array}$ & $\begin{array}{l}\text { Profundidad } \\
\text { y tipo de } \\
\text { sedimento }\end{array}$ & $\begin{array}{l}\text { Localidad } \\
\text { tipo }\end{array}$ & $\begin{array}{l}\text { Inserción del } \\
\text { filamento } \\
\text { branquial }\end{array}$ \\
\hline $\begin{array}{l}\text { C. chilensis Hartmann-Schröder, } 1965 \\
\text { Este estudio }\end{array}$ & $22-26$ & $\begin{array}{c}3 \\
\text { largos }\end{array}$ & $\begin{array}{c}1.703 \\
0,5 \text { por }>16 \text { por }>75\end{array}$ & $\begin{array}{l}50-160 \mathrm{~m}, \\
\text { mixto }\end{array}$ & Chile & $\begin{array}{l}\text { Parte anterior } \\
\text { del setígero } 3\end{array}$ \\
\hline C. abyssalis Hartman, 1967 & - & - & $\begin{array}{c}9 \\
0,5 \text { por }>4 \text { por }>23\end{array}$ & $3.655 \mathrm{~m}$ & Chile & $\begin{array}{l}\text { Parte anterior } \\
\text { del setígero } 3\end{array}$ \\
\hline C. alba Hartman, 1967 & - & - & $\begin{array}{c}1 \\
1,5 \text { por }>16 \text { por }>23\end{array}$ & $957 \mathrm{~m}$ & Chile & segmento $4-5$ \\
\hline C. heterochaeta Orensanz, 1976 & - & - & $\begin{array}{c}4 \\
\text { ? por }>9 \text { por }>37\end{array}$ & $\begin{array}{l}150-310 \mathrm{~m} \\
\text { mixto }\end{array}$ & Argentina & $\begin{array}{l}\text { Parte posterior } \\
\text { del setígero } 2\end{array}$ \\
\hline C. laeviseta Hartmann-Schröder, 1962 & $14 ?$ & $\begin{array}{c}3 \\
\text { cortos }\end{array}$ & $\begin{array}{c}13 \\
0,4 \text { por }>2,5 \text { por }>21\end{array}$ & $7 \mathrm{~m}$ & Perú & $\begin{array}{l}\text { Parte anterior } \\
\text { del setígero } 3\end{array}$ \\
\hline
\end{tabular}

A: Ancho en milímetros, L: Largo en milímetros

1Juan I. Cañete. Universidad de Magallanes, Punta Arenas, Chile. 


\section{Agradecimientos}

Los autores agradecen al proyecto FONDECYT de Iniciación $\mathrm{N}^{\circ} 11121487$ por el financiamiento otorgado para realizar la presente investigación. En segundo lugar agradecemos al Dr. Eduardo Quiroga, Biol. Mar. Guillermo Alarcón e Iván Salinas, Téc. Francisco Gallardo y Sr. Benjamín Ganga por el apoyo en terreno para la obtención de los individuos examinados. Además agradecemos a René Astudillo por la preparación de las figuras y a Fernando Jiménez por el análisis granulométrico. También al Departamento de Física de la Universidad Técnica Federico Santa María en Valparaíso por la Microscopía Electrónica de Barrido. Finalmente se agradece a los tres evaluadores que aportaron mejoras y constructivas observaciones al presente artículo.

\section{LITERATURA CITADA}

Blott SJ \& K Pye. 2001. Gradistat: a grain size distribution and statistics package for the analysis of unconsolidated sediments. Earth Surface Processes and Landforms (26): 1237-1248.

Carrasco F. 1977. Polychaeta (Annelida) de Bahía de Concepción, Chile. Familias Orbiniidae, Cirratulidae, Cossuridae, Capitellidae y Ampharetidae, con la descripción de tres especies y una subespecie nuevas. Boletín de la Sociedad de Biología de Concepción 51(1): 67-92.

Day J. 1963. The polychaete fauna of South Africa. Part. 8: New species and records from grab samples and dredgins. Bulletin of the British Museum (Natural History), Zoology 10:383-445.

Ewing RM. 1984. Family Cossuridae Day, 1963. In: Uebelacker JM \& PG Johnson (eds). Taxonomic guide to the polychaetes of the northern Gulf of Mexico Vol. 1: 4.1-4.6. Barry A. Vittor \& Associates, Mobile.

Fournier J \& M Petersen. 1991. Cossura longocirrata: Redescription and distribution, with notes on reproductive biology and a comparison of described species of Cossura (Polychaeta: Cossuridae). Ophelia Supplement 5: 63-80.

Gallardo VA, JG Castillo \& LA Yánez. 1972. Algunas consideraciones preliminares sobre la Ecología bentónica de los fondos sublitorales blandos en la Bahía de Concepción. Boletín, Sociedad de Biología de Concepción 44: 169-190.

Gallardo V, M Palma, F Carrasco, D Gutiérrez, L Levin \& J Cañete. 2004. Macrobenthic zonation caused by the oxygen minimum zone on the shelf and slope off central Chile. Deep-Sea Research II 51: 2475-2490.

Gardiner S \& W Wilson. 1979. New records of polychaete annelids from North Carolina with the description of a new species of Sphaerosyllis (Syllidae). Journal of the Elisha Mitchell Scientific Society 93: 159-172.
Gutiérrez D, V Gallardo, S Mayor, C Neira, C Vásquez, J Sellanes, M Rivas, A Soto, F Carrasco \& M Baltazar. 2000. Effects of dissolved oxygen and fresh organic matter on the bioturbation potential of macrofauna in sublittoral sediments off central Chile during the 1997/1998 El Niño. Marine Ecology Progress Series 202: 81-99.

Gutiérrez D, L Quipúzcoa, E Enríquez \& R Marquina. 2003. El macrobentos y las condiciones ambientales en otoño 2003: ¿Hay evidencias de variación interanual significativa desde el 2000? Informe IMARPE, Perú 38(2): 229-235.

Hartman O. 1955. Endemism in the North Pacific Ocean, with emphasis on the distribution of marine annelids, and descriptions of new or little known species. In: Essays in the Natural Sciences in honor of Captain Allan Hancock, University of Southern California, pp. 39-60.

Hartman O. 1967. Polychaeta annelids collected by the USNS Eltanin and Staten Island Cruises, chiefly from Antarctic Seas. Allan Hancock Monographs in Marine Biology 2: 1387.

Hartmann-Schröder G. 1965. Die Polychaeten des Sublitorals. In: Hartmann-Schröder G \& G Hartmann (eds). Zur Kenntnis des Sublitorals der chilenischen Küste unter besonderer Berücksichtigung der Polychaeten und Ostracoden. (Mit bemerkungen über den Einfluss sauerstoffarmer Strömungen auf die Besiedlung von marien Sedimenten). Mitteilungen aus dem Hamburgischen Zoologischen Museum und Institut 62:59-305.

Hilbig B. 1996. Family Cossuridae Day, 1963. In: Blake JA, B Hilbig \& PH Scott (eds). Taxonomic atlas of the benthic fauna of the Santa Maria Basin and Western Santa Barbara Channel. Vol. 6. The annelida. Part 3. Polychaeta: Orbiniidae to Cossuridae, pp. 385-404. Santa Barbara Museum of Natural History Publications, Santa Barbara.

Jones ML. 1956. Cossura pygodactylata, a new annelid from San Francisco Bay (Polychaeta: Cirratulidae). Journal of the Washington Academy of Sciences 46(4): 127-130.

Liñero-Arana I \& O Díaz-Díaz. 2010. A new species of Cossuridae (Annelida: Polychaeta) from Venezuela. Interciencia 35(10): 789-792.

Oyarzún C, F Carrasco \& V Gallardo. 1987. Some characteristics of macrobenthic fauna from the organicenriched sediments at Talcahuano, Chile. Cahiers de Biologie Marine 28: 429-446.

Read G. 2000. Taxonomy and distribution of a new Cossura species (Annelida: Polychaeta: Cossuridae) from New Zealand. Proceedings of the Biological Society of Washington 113(4): 1096-1110.

Read G \& K Fauchald. 2016. World Register of Polychaeta. <http:// http://www.marinespecies.org/polychaeta/ aphia.php?p=taxdetails\&id=326848 > 
Reish D. 1958. Description of a new species of Cossura (Annelida: Polychaeta) from Mississippi delta. Journal of the Washington Academy of Sciences 48: 53-55.

Rozbaczylo N. 1985. Los anélidos poliquetos de Chile. Índice sinonímico y distribución geográfica de especies. Monografías Biológicas 3: 1-284. Pontificia Universidad Católica de Chile, Santiago.

Sellanes J, E Quiroga, C Neira \& D Gutiérrez. 2007. Changes of macrobenthos composition under different ENSO cycle conditions on the Continental Shelf off central Chile. Continental Shelf Research 27: 1002-1016.

Soto E, E Quiroga, B Ganga \& G Alarcón. 2016. Influence of organic matter inputs and grain size on soft-bottom macrobenthic biodiversity in the upwelling ecosystem of central Chile. Marine Biodiversity, April 2016: 1-18. <doi 10.1007/s12526-016-0479-0>

Thulin G. 1921. Biologisch-faunistische Untersuchungen aus dem Öresund. IV. Über Cossura longocirrata Webster und Benedict und über die Röhren von Disoma multisetosum Örsted. Lunds Universitets Arsskrift, N.F., Avd. 2, 17(10): 3-7.

Turner RD \& PJ Boyle. 1975. Studies of bivalve larvae using the scanning electron microscope and critical point drying. Bulletin of the American Malacological Union 40: 59-65.
Tzetlin A. 1994. Fine morphology of the feeding apparatus of Cossura sp. (Polychaeta, Cossuridae) from the White Sea. In: Dauvin JC, L Laubier \& DJ Reish (eds). Actes de la 4ème Confèrence Internacionale des Polychètes. Mémoires du Muséum National d'Histoire Naturelle, Paris 162: 137143.

Webster H \& J Benedict. 1887. The Annelida Chaetopoda from Eastport, Maine. Annual Report of the United States Commission of Fish and Fisheries, Washington, pp. 707-755. [Appendix D.12]

Wu B \& M Chen. 1977. Heterocossura, a new genus of Cossuridae (Polychaeta: Sedentaria). Acta Zoologica Sinica 23:97-101

Zhadan A. 2015. Cossuridae (Annelida: Polychaeta: Sedentaria) from Australian and adjacent waters: the first faunistic survey. Records of the Australian Museum 67(1): 1-24.

Zhadan A, E Vortsepneva \& A Tzetlin. 2012. Redescription and Biology of Cossura pygodactylata Jones, 1956 (Polychaeta: Cossuridae) in the White Sea. Invertebrate Zoology 9(2): 115-125.

Recibido el 6 de junio de 2016 y aceptado el 23 de septiembre de 2016

Editor: Claudia Bustos D. 